\title{
Mechanisms for polycrystalline silicon defect passivation by hydrogenation in an electron cyclotron resonance plasma
}

\author{
E. S. Cielaszyk, K. H. R. Kirmse, R. A. Stewart, and A. E. Wendt ${ }^{\text {a) }}$ \\ Engineering Research Center for Plasma-Aided Manufacturing, University of Wisconsin-Madison, Madison, \\ Wisconsin 53706-1691
}

(Received 17 August 1995; accepted for publication 25 September 1995)

\begin{abstract}
An in-line mass spectrometer and Langmuir probes have been employed to examine mechanisms of plasma immersion hydrogen passivation of grain boundary defects in polycrystalline silicon thin film transistors. Relative fluxes of $\mathrm{H}^{+}$and $\mathrm{H}_{2}^{+}$as well as total ion current density were measured at the substrate location in an electron cyclotron resonance hydrogen discharge. Measurements were made over a range of operating conditions over which passivation rates have been shown to vary dramatically. Data presented show a strong correlation of both $\mathrm{H}^{+}$flux and ion bombardment energy with good transistor performance obtained at operating pressures below 1 mTorr. This suggests that discharge operating conditions that promote dissociation of $\mathrm{H}_{2}$ to form $\mathrm{H}$ and $\mathrm{H}^{+}$(which may diffuse more rapidly through solid material than $\mathrm{H}_{2}$ ), as well as increased sheath voltages and therefore ion energy at the substrate, are important to obtaining acceptable process rates. (C) 1995 American Institute of Physics.
\end{abstract}

Acceptable electrical performance of polysilicon thin film transistors (TFTs) for several applications, including driver circuitry for active matrix liquid crystal displays (AMLCD), requires the hydrogen passivation of defect sites in the polycrystalline silicon grain boundaries. ${ }^{1,2}$ One of the most promising methods for passivation is exposure of the fabricated transistors to low pressure $(<1$ mTorr) hydrogen plasmas generated in an electron cyclotron resonance (ECR) source. However, ECR-based reactors may not be well suited for large area substrate processing under development for AMLCD flat panel displays. To aid in the design of processes based on more suitable reactor types employing, for example, inductively coupled or helicon plasmas, it is helpful to understand the basic mechanisms responsible for efficient plasma exposure hydrogenation. Specifically, we address the hypothesis that high passivation rates observed in low pressure ECR hydrogen discharges are the result of enhanced dissociation of $\mathrm{H}_{2}$ at discharge pressures below 1 mTorr. ${ }^{3}$ It is believed that because atomic hydrogen diffuses through solid material more readily than molecular hydrogen, conditions that favor a high flux of either neutral or ionized atomic hydrogen to the substrate will result in high rates of hydrogen passivation.

To investigate these mechanisms, we examine plasma conditions in a $\mathrm{H}_{2}$ ECR discharge identical to that used in a study of polysilicon passivation, over a range of operating parameters over which passivation effectiveness was found to vary dramatically. ${ }^{1}$ This study involves examination of the mass distribution and flux of ions, but not neutrals, incident on the substrate. There are practical reasons for studying only the ions, and it is believed that the ion data on its own provides substantial support for the significance of the role of atomic hydrogen. First, the presence of atomic hydrogen ions, $\mathrm{H}^{+}$, can be considered to be a good indicator of the presence of neutral atomic hydrogen. In addition, even if the

${ }^{\text {a)} E l e c t r o n i c ~ m a i l: ~ w e n d t @ e n g r . w i s c . e d u ~}$ concentration of $\mathrm{H}^{+}$ions is lower than that of neutral $\mathrm{H}$ atoms in the gas phase, the flux of $\mathrm{H}^{+}$ions may be higher due to acceleration of ions toward the substrate by electrostatic fields in the plasma. Furthermore, because of this acceleration, ions reach the surface with greater kinetic energy than the neutrals, making them more likely to adsorb on the surface once they reach it. ${ }^{4}$

Hydrogen plasmas were generated in an ASTeX S-1500i ECR source, described in detail elsewhere. ${ }^{5}$ Microwave power at $2.45 \mathrm{GHz}$ entered the source through a quartz window, and reflected power was minimized by a three-stub tuner matching system. Hydrogen was introduced at the source region through a gas feed ring. Pressures reported were measured "downstream" in the chamber near the substrate location with a capacitance manometer (MKS Baratron Type 390HA). Pressures were also measured with a nitrogencalibrated ion gauge with a hydrogen sensitivity conversion factor of 0.44. The ion gauge was used for comparison with conditions in the transistor hydrogenation study ${ }^{1}$ and because the pressure range of interest here is outside the optimal range for either gauge. In this pressure range, the capacitance manometer results remained a constant factor of 8.6 greater than the applicable ion gauge measurements. The start-up procedure included maintaining a hydrogen plasma at $600 \mathrm{~W}$ and 0.25 mTorr for $30 \mathrm{~min}$ to "season" the chamber. The top and bottom ECR electromagnet currents were held at 185.6 and 113.6 A, respectively.

Relative atomic and molecular hydrogen ion fluxes were measured by a magnetically shielded, in-line UTI 100-C quadrupole mass spectrometer described in detail elsewhere. ${ }^{6}$ The mass spectrometer was mounted inside a differentially pumped housing system that replaced the substrate holder. Particles enter the spectrometer from the plasma through a $150 \mu \mathrm{m}$ diameter pinhole in a disk of nickel foil. The plane of the foil is located in the reactor in the axial position corresponding to the substrate location. Ion fluxes were measured over a discharge power range of 300-900 W and for $\mathrm{H}_{2}$ pressures between 0.25 and 2.25 mTorr. Experiments 
were conducted in nonincremental order to minimize the possible influence of instrumental drift on trends observed in the data.

Ions pass unimpeded through the mass spectrometer, so the signal strength is directly proportional to the flux of ions to the nickel foil from the plasma. Neutrals do not contribute to the mass spectrometer signal in this case because the ionizer at the inlet of the spectrometer was absent for these experiments. When the spectrometer is configured to sample neutrals, there is ambiguity in the signal associated both with dissociation of molecular hydrogen in the ionizer and with the contribution to the signal from ions originating in the discharge.

Total ion flux was determined with a Langmuir probe ${ }^{7}$ at the pinhole location with the mass spectrometer removed. For these measurements, the substrate holder was in place with the wafer stage retracted a few centimeters to prevent interference with the probe measurements. The one-sided 0.125 in. diameter planar probe was directed facing the top of the ECR system, in a plane perpendicular to the static magnetic field. Measurements were made in nonincremental order over a pressure range of $0.25-2.25$ mTorr with constant input power of $600 \mathrm{~W}$.

Langmuir probe current-voltage $(I-V)$ characteristics were analyzed using conventional techniques to determine plasma and floating potentials, $\varphi_{p}$ and $\varphi_{f}$, and ion saturation current density. Plasma potential was determined as the probe bias voltage at which $d I / d V$ is a maximum. The probe floating potential $\varphi_{f}$ determined as the probe bias voltage for which the probe current is zero, is expected to be equal to the electrostatic potential on the surface of an unbiased substrate. The ion contribution to the probe current was found by a fit to the $I-V$ curve at large negative bias voltages. This fit was extrapolated to the plasma potential to obtain the ion saturation current density.

The mass spectra of ion fluxes at the substrate location were measured over a range of hydrogen pressures $(0.25-$ $2.25 \mathrm{mTorr})$ and discharge powers $(300-600 \mathrm{~W})$. Peaks in the spectra were observed for masses corresponding to $\mathrm{H}^{+}$ and $\mathrm{H}_{2}^{+}$only $\left(\mathrm{H}_{3}^{+}\right.$, which is likely to be present at higher discharge pressure, was not observed). Relative ion fluxes reported are peak heights obtained from the mass spectra.

The variation of $\mathrm{H}^{+}$and $\mathrm{H}_{2}^{+}$fluxes versus pressure at a fixed power $(600 \mathrm{~W})$ is shown in Fig. 1(a). A significant increase in the flux of $\mathrm{H}_{2}^{+}$and decrease in the flux of $\mathrm{H}^{+}$was observed as the pressure was increased over this range. Because the sensitivity of the mass spectrometer varies with mass, we cannot compare peak heights for $\mathrm{H}^{+}$and $\mathrm{H}_{2}^{+}$with one another on an absolute scale, but it is meaningful to examine their variation relative to one another as conditions are varied. Thus, the ratio of the peak heights for $\mathrm{H}^{+}$and $\mathrm{H}_{2}^{+}$is shown as a function of pressure in Fig. 1(b). This ratio varies by a factor of 6 over this pressure range, strongly correlating to observed variations in polysilicon hydrogenation rates, and consistent with the hypothesis that some combination of $\mathrm{H}$ and $\mathrm{H}^{+}$is responsible for hydrogenation.

Plasma conditions were monitored for the same range of discharge conditions with a planar Langmuir probe. Langmuir probe $I-V$ curves were fairly noisy under the low pres-

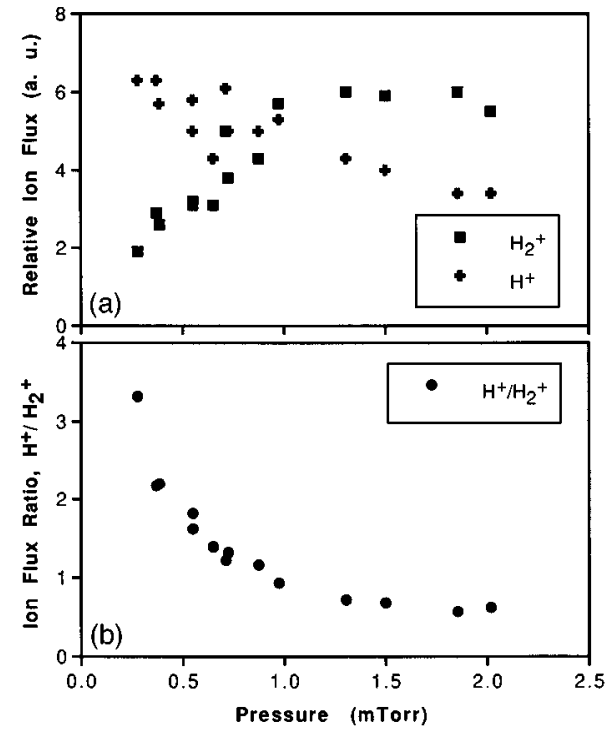

FIG. 1. Relative ion flux vs pressure as measured by mass spectrometer peak heights. The input power was held constant at $600 \mathrm{~W}$. (a) Individual relative ion flux for atomic and molecular species. (b) The relative ion flux ratio of $\mathrm{H}^{+} / \mathrm{H}_{2}^{+}$.

sure conditions studied here, resulting in appreciable scatter in ion current density and potential values, and averages from several traces are shown in Figs. 2 and 3, respectively. Because the variation of ion saturation current density with pressure has the same shape as that of the relative $\mathrm{H}_{2}^{+}$flux and differs from that of the relative $\mathrm{H}^{+}$flux, it is concluded that $\mathrm{H}_{2}^{+}$is the dominant ion in the system over the range of conditions studied. So although the flux of $\mathrm{H}^{+}$increases as pressure is reduced, it is unlikely that $\mathrm{H}^{+}$flux exceeds $\mathrm{H}_{2}^{+}$ flux under any of the conditions studied.

Another pressure dependent parameter that may play an important role in the effectiveness of this process is the energy of the ions impacting the surface. Ion impact energy can

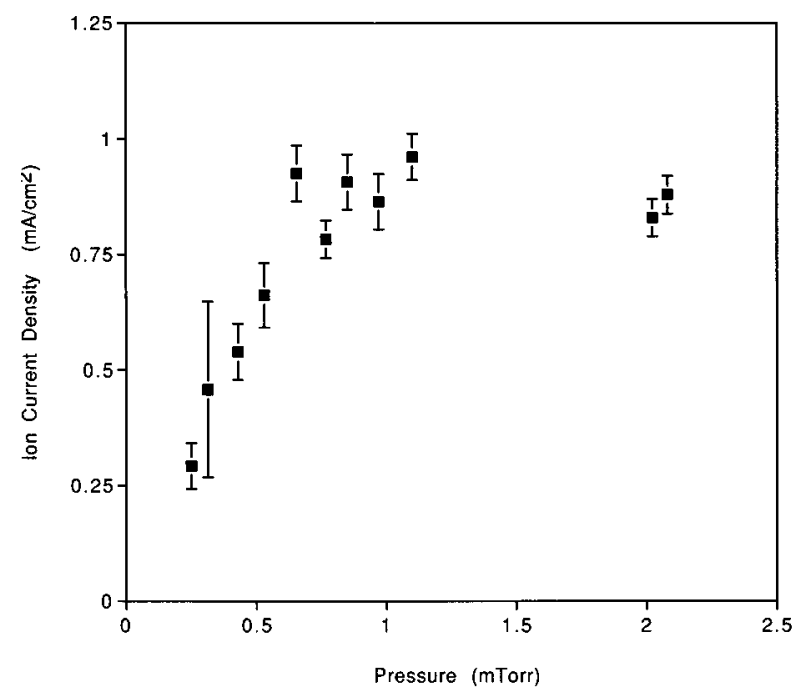

FIG. 2. Ion current density vs pressure as calculated from Langmuir probe curves. The input power was held constant at $600 \mathrm{~W}$. Data points shown are averages over several measurements, with error bars representing the standard deviation of the mean. 


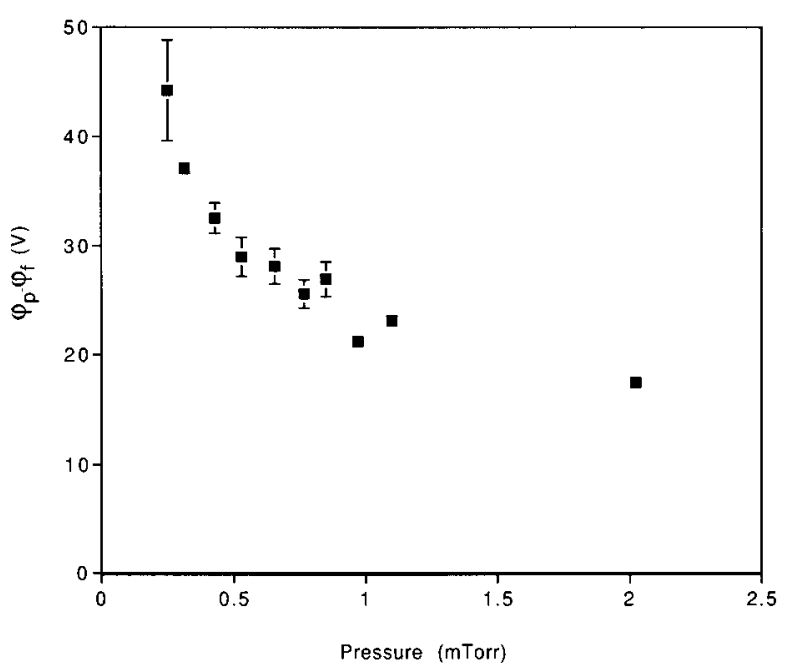

FIG. 3. The difference between the plasma potential $\varphi_{p}$ and the floating potential $\varphi_{f}$ vs pressure as calculated from Langmuir probe curves. The input power was held constant at $600 \mathrm{~W}$. Data points shown are averages over several measurements, with error bars representing the standard deviation of the mean.

be estimated from Langmuir probe data as $E_{i}=e\left(\varphi_{p}-\varphi_{f}\right)$, where $\varphi_{p}$ and $\varphi_{f}$ are the plasma potential and floating potentials, respectively. The potential difference $\varphi_{p}-\varphi_{f}$, as shown in Fig. 3 as a function of pressure, establishes itself across the sheath at the boundary between the plasma and the substrate surface, setting up an electric field that accelerates ions into the surface. Our measurements indicate a signifi- cant increase in ion bombardment energy with the reduction in pressure from 2.25 to 0.25 mTorr.

As $\mathrm{H}_{2}$ pressure in the ECR discharge is decreased, we observe an increase in flux of $\mathrm{H}^{+}$to the surface, although the total ion flux is decreasing. The flux of neutral $\mathrm{H}$ atoms is likely to have a concomitant increase, enhancing the process rate. In addition, the kinetic energies of both ionic species reaching the substrate increase with decreasing pressure, an effect that is likely to contribute to the enhanced process rates observed at lower pressures. $\mathrm{H}^{+}$ions with higher energy are more likely to stick to the substrate surface and become available for diffusion, and in addition, energetic $\mathrm{H}_{2}^{+}$ions may dissociate on impact, generating even more atomic hydrogen.

This work is supported by the National Science Foundation under Grant No. EEC-8721545.

${ }^{1}$ R. A. Ditizio, S. J. Fonash, and B.-C. Hseih, J. Vac. Sci. Technol. A 10, 59 (1992)

${ }^{2}$ R. A. Ditizio, G. Liu, S. J. Fonash, B.-C. Hseih, and D. W. Greve, Appl. Phys. Lett. 56, 1140 (1990).

${ }^{3}$ G. Liu and S. J. Fonash, Appl. Phys. Lett. 62, 2554 (1993).

${ }^{4}$ N. H. Nickel, W. B. Jackson, I.-W. Wu, C.-C. Tsai, and A. Chiang, Phys. Rev. B 52, 7791 (1995).

${ }^{5}$ J. A. Meyer, K. H. R. Kirmse, J.-S. Jeng, S. Y. Perez-Montero, H. L. Maynard, A. E. Wendt, J. W. Taylor, and N. Hershkowitz, Appl. Phys. Lett. 64, 15 (1994).

${ }^{6}$ K. H. R. Kirmse, A. E. Wendt, S. B. Disch, J. Z. Wu, I. C. Abraham, J. A. Meyer, R. A. Breun, and R. C. Woods, J. Vac. Sci. Technol. (to be published).

${ }^{7}$ N. Hershkowitz, in Plasma Diagnostics Volume 1-Discharge Parameters and Chemistry, edited by O. Auciello and D. L. Flamm (Academic, San Diego, CA, 1989), p. 113. 